\title{
QoS controlled capacity offload optimization in heterogeneous networks
}

\author{
Siva Priya Thiagarajah, Mohamad Yusoff Alias, Wooi-Nee Tan \\ Faculty of Engineering, Multimedia University, Malaysia
}

\begin{tabular}{l} 
Article Info \\
\hline Article history: \\
Received Feb 15, 2020 \\
Revised Apr 24, 2020 \\
Accepted Jun 20, 2020 \\
\hline Keywords: \\
Branch and bound \\
Capacity maximization \\
LTE \\
Optimization \\
QoS control \\
Resource management
\end{tabular}

\begin{abstract}
An efficient resource allocation mechanism in the physical layer of wireless networks ensures that resources such as bandwidth and power are used with high efficiency in spite of low delay and high edge user data rate. Microcells in the network are typically set with bias settings to artificially increase the Signal-to-Interference-Plus-Noise Ratio, thus encouraging users to offload to the microcell. However, the artificial bias settings are tedious and often suboptimal. This work presents a low complexity algorithm for maximization of network capacity with load balancing in a heterogeneous network without the need for bias setting. The small cells were deployed in a grid topology at a selected distance from macrocell to enhance network capacity through coverage overlap. User association and minimum user throughput were incorporated as constraints to enable closer simulation to real word Quality of Service requirements. The results showed that the proposed algorithm was able to maintain less than $10 \%$ user drop rate. The proposed algorithm can increase user confidence as well as maintain load balancing, maintain the scalability, and reduce power consumption of the wireless network.
\end{abstract}

This is an open access article under the CC BY-SA license.

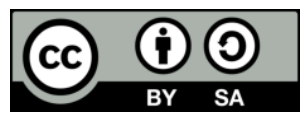

\section{Corresponding Author:}

Siva Priya Thiagarajah,

Faculty of Engineering,

Multimedia University,

63100, Cyberjaya, Malaysia.

Email: siva.priya.thiagarajah@mmu.edu.my

\section{INTRODUCTION}

Fifth Generation $(5 \mathrm{G})$ networks are promising significantly higher mobile data volume per area, increased volumes of connected devices, higher user data rate, increased battery life, and reduced end-to-end latency [1]. Experts agree that one way to achieve 5G capacity and coverage goals is to deploy heterogenous networks (HetNet). HetNets are deployed using either the single or multiple Radio Access Technology (RAT) at the different tiers. A multiple RAT 2-tier HetNet may use long term evolution (LTE) technology in the macrocell, and use WiFi in the small cell tier [2]. Alternatively, single RAT HetNet may deploy LTE on both the macro and small cells. Single RAT offloading uses the same time frequency resources as the macrocells, thus increasing spectrum efficiency [3, 4]. Single RAT offloading can be done with less signalling overhead, reduced delay for delay sensitive data and seamless mobility [5]. It also has a disadvantage of intracellular interference but this can be managed by using advanced interference management techniques [6].

HetNets may deploy cell range expansion (CRE) [7] to manage interference as well as to extend its coverage area. Intracell interference in a HetNet is mainly caused by the transmission power of the macrocell, which causes interference to the small cell in the downlink. The macrocell also induces more users to attach 
themselves to it, since it promises more transmission gains to overcome path loss. The bias concept was proposed to overcome transmit power imbalances and to achieve better load distribution between macrocells and small cells in a HetNet. A positive range expansion bias is added to the small cell's downlink pilot signal strength to increase signal to interference plus noise ratio (SINR) and attract users to attach themselves to it [8]. This will force users to connect to the nearest small cell instead of the higher powered macrocell. At the macrocell coverage edge, the use of CRE can expand the coverage of the HetNet.

Optimization in wireless communication is the process of meeting the objective function for a given scenario of users and base station layout,with resource constraints such as power, bandwidth and delay [9] . The best possible use of the resources is ensured by optimizing a certain criteria or objective, for example maximizing the edge user throughput or minimizing the base station transmit power. Optimization in resource allocation of radio frequency is often a non-deterministic polynomial (NP) hard and sometimes a non-convex [10] problem. The objective function can either be linear or non-linear, with either linear and/or non-linear constraints. Branch and bound (BB) is a popular algorithm used in the radio resource allocation of HetNet [11-15]. It uses node fathoming and infeasibility pruning to reduce the time taken to arrive at an optimal solution, thus giving it a higher efficiency than the exhaustive search to find an optimum solution [8]. Node fathoming is done when the node meets the constraints but cannot get a lower value of the objective function at any node that descends from it. A branch is pruned or further search within the subset is stopped to save storage space when the output of the branch is higher than the bounding value of incumbent objective function. Recent advances in CRE based research is steering to find the optimal bias value through the use of optimization techniques.

Yanzan Sun et al. [16] worked to estimate the upper bound for a bias for the pico cells that were overlaid on a macrocell to achieve target SINR for users while maintaining a good load balance. The discussion in [17] highlighted that it is difficult to prescribe the optimal bias value by leveraging optimization techniques, especially in co-channel deployments when macrocells and small cells are deployed in the same frequency band. The use of bias in the co-channel in reality increases interference; because the user is actually close enough to the macrocell for the macrocell to be a strong source of interference. This leads to argument that using a bias may not actually solve the issue of interference mitigation. S. Nathaniel et al. [18] consider multi criteria optimization using weight associated normalized values for cell power consumption, service tier and user velocity. Capacity in a cell is offloaded when a predefined capacity percentage of that cell has been occupied.

The work in [19] optimizes user-cell association in a video uploading scenario, and evaluates the performance of radio resource strategies in the uplink for a pico-macro HetNet. While agreeing with past works that small cell placement along the edge of coverage can increase system capacity, the work in [20] also suggests that small nodes placed closer to the macro base station may exploit a trade-off between coverage and a macro node to relay node link performance. The idea of small cell node placement adopted in this work, is inspired by [20]. The work in [21] takes into account the user behaviors and its association with the cells in a HetNet and exploits it to maximize long- term expected system throughput. The authors in [22] designed a system level simulator for the study of cell offload in HetNets with small cells being placed at predetermined locations from the macrocell. The work proved that aggregated throughput can be maximized when macro base stations are highly offloaded and propose the use of biases to offload the macros and achieve user fairness. One of the main concerns in HetNet deployment is the optimized usage of the available resources such as bandwidth. The user experience, gauged through the received throughput, is a critical determinant in the Quality of Service (QoS) provided by the mobile operator. One metric to measure QoS is the mean user throughput [23]. To the best of our knowledge, most resource management algorithms do not consider the minimum user throughput as a factor to maintain the link connection's QoS. The contribution of this work is therefore:

- to take into consideration the QoS of the user through setting of a minimum user throughput as a bounding factor in the optimization.

- to not use a bias setting for small cells, thus providing a realistic SINR scenario in which the user attachment is optimized.

The rest of this paper is structured as follows: the problem formulation, system modelling and proposed algorithms are in section 2 while section 3 discusses the results and findings of the work. Section 4 concludes the paper.

\section{PROBLEM FORMULATION}

\subsection{Assumptions and modelling}

The HetNet consists of K base stations; V macrocells transmitting at $43 \mathrm{dBm}$ each and $\mathrm{W}$ small cells transmitting at $23 \mathrm{dBm}$ each. The macrocell has a cell radius of $500 \mathrm{~m}$ and the small cell radius is $50 \mathrm{~m}$ [24]. 
Each small cell is situated at 50m inter site distance (ISD) from the macrocell. The total users in the HetNet are denoted by $U$ where any user can attach to the macro or small cell as allocated by the algorithm. The desired user is denoted by $u$. The small cell or macrocell that $u$ is attached to is denoted by $k$, otherwise known as the desired base station. Only one resource block (RB) per cell is considered. The RB uses time division multiple access (TDMA) whereby one time slot is considered for each RB; for every timeslot, we consider a maximum of $\mathrm{F}$ resource elements (RE). The maximum bandwidth available per cell is denoted by $B_{k}$. Each RE assumes binary phase shift keying (BPSK) modulation, such that each RE will allocate $1 \mathrm{bit} / \mathrm{s}$ after the optimization to the user attached to it.

The simulation considers the downlink scenario and assumes flawless synchronization between LTE cells such that there is no intracellular interference. The channel noise conditions between user and cells are assumed to be additive white gaussian noise (AWGN) distributed. Intercellular interference is considered to be SINR such that interference occurs only if the same RE is reused by two different cells (macrocell or small cell) in the immediate adjacent tier. The SINR of user $u$ when served by cell $k$ and using RE $f$ is expressed as [25]

$$
\operatorname{SINR}_{u k f}=\frac{x_{u k f} P_{u k f} G_{u k f}}{\sum_{\substack{i=1 \\ i \neq u}}^{U} \sum_{\substack{k=1 \\ k \neq j}}^{K} x_{i j f} P_{i j f} G_{i j f}+n(t)}
$$

where $P_{u k f}$ denotes the transmitted power of cell $k$ to user $u$. $G_{u k f}$ denotes the link gain from cell $k$ to user $u$ over RE $f . n(t)$ is the AWGN noise. $x_{u k f}$ is the user association variable when desired user $u$ is connected to cell $k$ on subband $f . x_{i j f}$ is the user association variable for other users who use the same subband $f$ on the other cells. $P_{i j f}$ and $G_{i j f}$ are received power and link gains associated with user variables $x_{i j f}$ respectively.

Subband allocation to the user can be non-contiguous. Both shadowing loss and Rayleigh fading are factored into the path loss modelling. The pathloss for each RE is calculated based on user channel gain condition and is modelled as (1) for macrocell and (3) for the small cells [21].

$$
\begin{aligned}
& L_{\text {macro }}=128.1+37.6 \log \left(d_{u}\right) \\
& L_{\text {smallcell }}=140.7+37.6 \log \left(d_{u}\right)
\end{aligned}
$$

where $L_{\text {macro }}$ and $L_{\text {smallcell }}$ are the path loss for the macrocell and the small cell, respectively in $\mathrm{dB}$, while $d_{u}$ is the distance between cell $k$ and user $u$ in $\mathrm{km}$.

Figure 1 shows the macrocell, small cell and users layout for the simulation. Up to $K=4$ is considered in the simulation, and maximum users, $U$, simulated are 24 . The user locations are kept constant as the number of $K$ is increased. $F=8$ subbands are assumed per cell. The aim of the work is to show proof of concept on how the different algorithms perform in the resource allocation, and thus, only 24 users and 8 subbands per cell are used in the simulations. The proposed model can be used to optimize any number of $K, U$ or $F$, with a restriction that $F$ must be increased in form of $2^{r}$ where $r \in\{1,2, \ldots, R\}$.

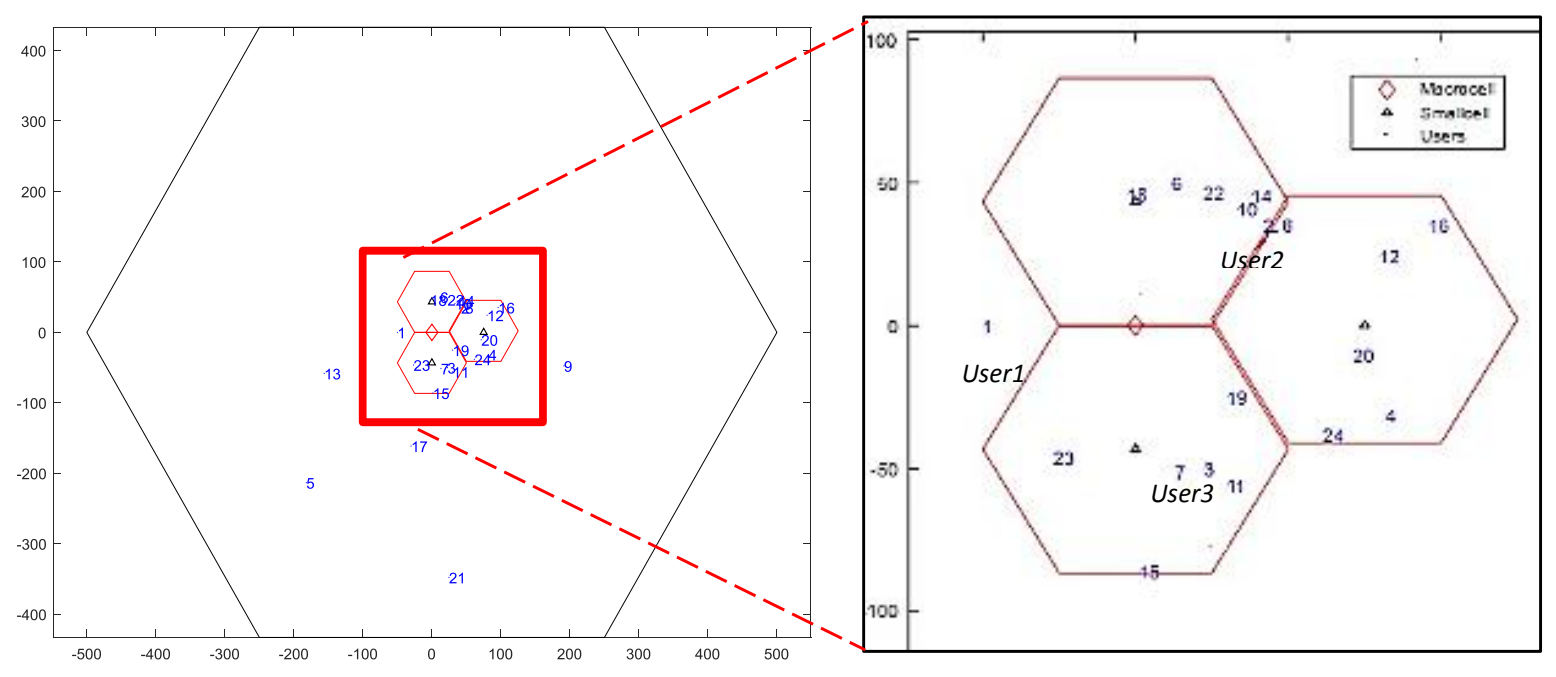

Figure 1. Proposed model 
Figure 2 shows the flow chart of the optimization flow for one run. In the first stage of the optimization, the user's distance is used to determine if association with a cell is possible. The user $u$ is associated with a cell $k$ if where $L_{u k}$ is either $L_{\text {macro }}$ or $L_{\text {smallcell }}$ and $P_{k}$ is the cell transmit power. The min value is pre-determined by the operator and is the threshold received power required at the user terminal to ensure that a link connection can be established between the user and the cell. where $C_{u k f}$ denotes the capacity of user $u$ when attached to cell $k$ if

$$
\frac{P_{k}}{L_{u k}} \geq \min
$$

where $L_{u k}$ is either $L_{\text {macro }}$ or $L_{\text {smallcell }}$ and $P_{k}$ is the cell transmit power. The min value is pre-determined by the operator and is the threshold received power required at the user terminal to ensure that a link connection can be established between the user and the cell.

If (4) is satisfied, then the user association variable, $x_{u k f}$ for $f \in\{1,2, \ldots, F\}$ will be created where $x_{u k f} \in X$, and $X$ is the combination of $x_{u k f}$ which provides the maximized HetNet capacity, z. Figure 2 also shows $z^{n}$, which is the maximized capacity stored by the optimizer. $z^{n}$ is an incumbent maximized value, and will be updated when a more maximized value is reached during the optimization process. The maximization of the HetNet overall capacity, which is the objective function, is defined as

$$
\max \quad z=\sum_{u=1}^{U} \sum_{k=1}^{K} \sum_{f=1}^{F} B_{f} \log _{2}\left(1+\operatorname{SIN} R_{u k f}\right)
$$

where $\mathrm{z}$ denotes total capacity in the HetNet and $\mathrm{B}_{f}$ is the subband bandwidth defined at $180 \mathrm{kHz}$. The throughput of each user $u$ can be calculated separately, if desired, using

$$
C_{u k f}=\sum_{k}^{K} \sum_{f}^{F} B_{f} \log _{2}\left(1+S I N R_{u k f}\right) \quad \forall u \in\{1,2, \ldots, U\}
$$

where $C_{u k f}$ denotes the capacity of user $u$ when attached to cell $k$.

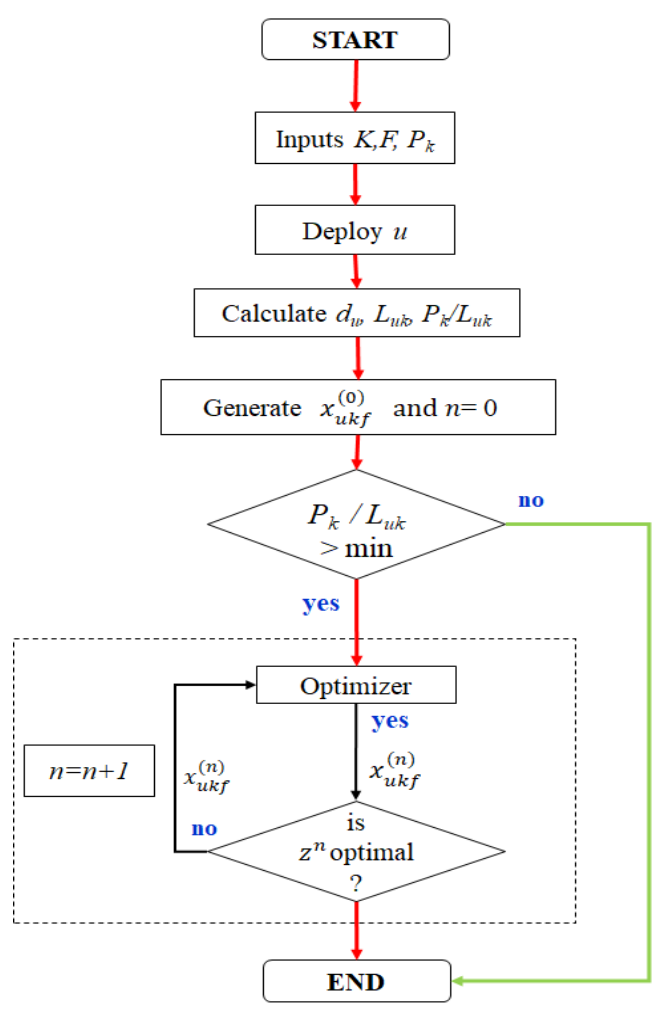

Figure 2. Optimization flowchart for a single run 


\subsection{Algorithm 1-user association constraints}

The user association is constrained by two factors. The first factor is that each user is only attached to one cell. The first factor is controlled by two constraint inequalities as described in (7) and (8).

$$
\begin{array}{ll}
\sum_{k}^{k} y_{u k}=1, & \forall u \in\{1,2, \ldots, U\} \\
\sum_{f}^{F} x_{u k f} \leq y_{u k} M, & \forall u \in\{1,2, \ldots, U\}, \quad \forall k \in\{1,2, \ldots, K\}
\end{array}
$$

where $M$ is an arbitrarily large number, $M>F$. For the simulations, $M$ is set as 1000 . The variable $y_{u k}$ is introduced to control the user association.

The second factor is that while the number of REs within cell $k$ that the user $u$ is attached to is not limited to one, an RE can only be leased to one user at any given timeslot. The second factor is expressed by:

$$
\sum_{u}^{U} x_{u k f} \leq 1 \quad \forall k \in\{1,2, \ldots, K\}, \quad \forall f \in\{1,2, \ldots, F\}
$$

Thus, the complete model for Algorithm 1 can be defined as:

$$
\max z=\sum_{u=1}^{U} \sum_{k=1}^{K} \sum_{f=1}^{F} B_{f} \log _{2}\left(1+\operatorname{SINR}_{u k f}\right)
$$

subject to:

$$
\begin{array}{ll}
\sum_{k}^{K} y_{u k}=1, & \forall u \in\{1,2, \ldots, U\} \\
\sum_{f}^{F} x_{u k f} \leq y_{u k} M, & \forall u \in\{1,2, \ldots, U\}, \quad \forall k \in\{1,2, \ldots, K\} \\
\sum_{u}^{U} x_{u k f} \leq 1, & \forall k \in\{1,2, \ldots, K\}, \quad \forall f \in\{1,2, \ldots, F\}
\end{array}
$$

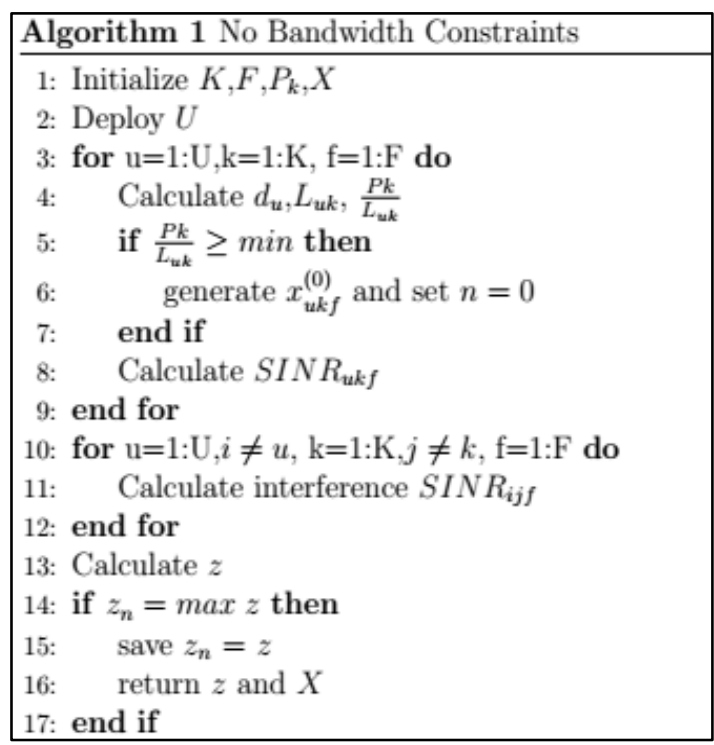

\subsection{Algorithm 2-minimum subband allocation constraint}

Algorithm 2 forces each new added user to be allocated a subband regardless of whether minimum QoS constraints are met. Along with the constraints in Algoritm1, the minimum subband algorithm introduces a new constraint, where each added user will need to be allocated at least one subband. This constraint is controlled by:

$$
\sum_{k}^{K} \sum_{f}^{F} x_{u k f} \geq 1, \quad \forall u \in\{1,2, \ldots, U\}
$$


Since $x_{u k f}$ is the user association parameter, (10) shows that each user $u$ must at least be attached to one subband $f$ from any but limited to one cell $k$.

The complete model for Algorithm 2 can be defined as:

$$
\max z=\sum_{u=1}^{U} \sum_{k=1}^{K} \sum_{f=1}^{F} B_{f} \log _{2}\left(1+S I N R_{u k f}\right)
$$

subject to:

$$
\begin{aligned}
& \sum_{k}^{K} y_{u k}=1, \quad \forall u \in\{1,2, \ldots, U\} \\
& \sum_{f}^{F} x_{u k f} \leq y_{u k} M, \quad \forall u \in\{1,2, \ldots, U\}, \quad \forall k \in\{1,2, \ldots, K\} \\
& \sum_{u}^{U} x_{u k f} \leq 1, \quad \forall k \in\{1,2, \ldots, K\}, \quad \forall f \in\{1,2, \ldots, F\} \\
& \sum_{k}^{K} \sum_{f}^{F} x_{u k f} \geq 1, \quad \forall u \in\{1,2, \ldots, U\}
\end{aligned}
$$

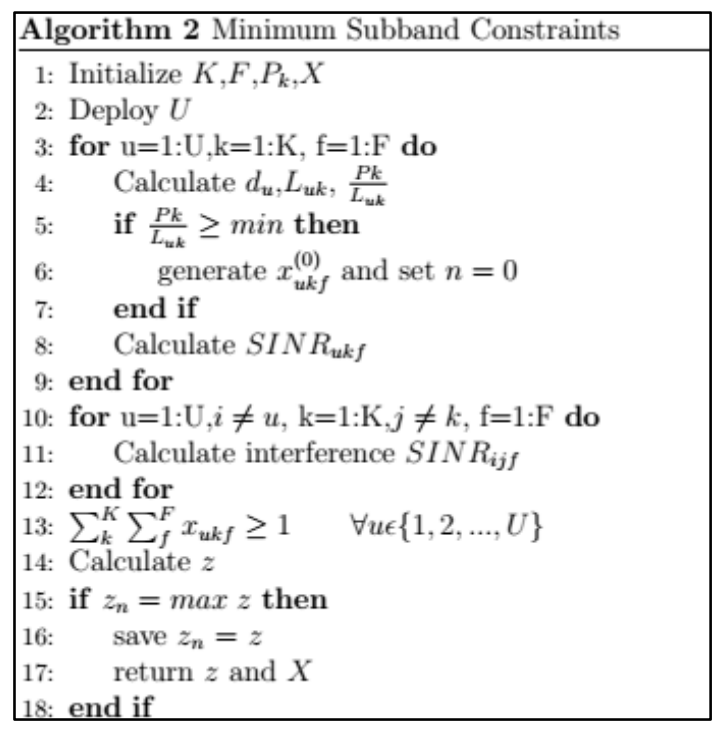

\subsection{Algorithm 3-minimum user throughput}

The minimum user throughput introduces an alternative constraint to (10), where each user must be allocated a subband. This algorithm controls the QoS by dropping users who do not meet the minimum constraint. In (6) is now used as a non-linear constraint and is re-expressed as:

$$
\sum_{k}^{K} \sum_{f}^{F} B_{f} \log _{2}\left(1+\operatorname{SINR}_{u k f}\right) \leq b(t) \quad \forall u \in\{1,2, \ldots, U\}
$$

where $b(t)$ is a value defined by the operator and defines the user downlink throughput. In this work, $b(t)$ is set at $180 \mathrm{kbps}$. The complete model for Algorithm 3 can therefore be defined as:

$$
\max z=\sum_{u=1}^{U} \sum_{k=1}^{K} \sum_{f=1}^{F} B_{f} \log _{2}\left(1+S I N R_{u k f}\right)
$$

subject to:

$$
\begin{aligned}
& \sum_{k}^{K} y_{u k}=1, \quad \forall u \in\{1,2, \ldots, U\} \\
& \sum_{f}^{F} x_{u k f} \leq y_{u k} M, \quad \forall u \in\{1,2, \ldots, U\}, \quad \forall k \in\{1,2, \ldots, K\} \\
& \sum_{u}^{U} x_{u k f} \leq 1, \quad \forall k \in\{1,2, \ldots, K\}, \quad \forall f \in\{1,2, \ldots, F\} \\
& \sum_{k}^{K} \sum_{f}^{F} B_{f} \log _{2}\left(1+S I N R_{u k f}\right) \leq b(t) \quad \forall u \in\{1,2, \ldots, U\}
\end{aligned}
$$




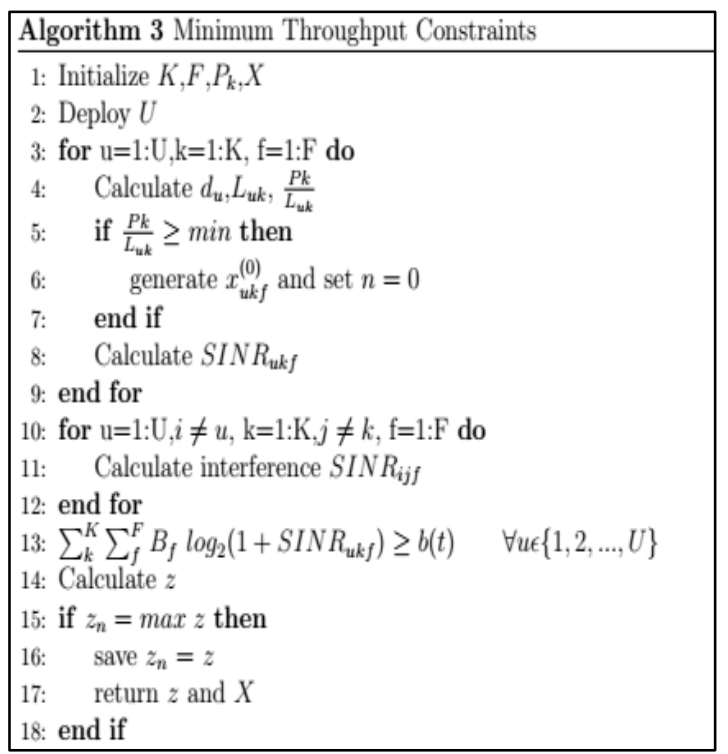

\section{RESULTS AND DISCUSSION}

\subsection{Outside small cell user performance/macro only users (User1)}

Throughout section 3, A1K2 indicates Algorithm 1 with $K=2$. Similarly, A2 and A3 indicate Algorithm2 and Algorithm3 respectively, while K3 and K4 represent $K=3$ and $K=4$ respectively. All three algorithms are based on BB. Figures 3(a)-3(c) show the performance of a typical user within the macrocell, but outside the small cell, also known as macro only user (Userl), with varying number of $K$ for $\mathrm{A} 1, \mathrm{~A} 2$ and A3. Userl's location is shown in Figure 1.

When $K=2$, Userl performance on A1 and A3 are comparable, with Userl experiencing between 12.5-22.5 Mbps even when up to 22 other users are added. In comparison, Userl on A2 experiences the lowest throughput as early as when 8 other users are added to the HetNet. As users are added, A3 outperforms with a better average User1 throughput of $18.9 \mathrm{Mbps}$, as compared to A2 at $10.15 \mathrm{Mbps}$ and A1 at $18.08 \mathrm{Mbps}$. At $K=3$, Figure 3(b) shows that A1 outperforms A3 in terms of Userl throughput, but A3 provides more stable Userl throughput over time as compared to A2. Figure 3(c) shows that at $K=4$, A3 performs the worst in terms of Userl average throughput. The results in Figures 3(a)-3(c) therefore show that when there is no offload constraint, increasing the small cells improves the throughput of macro only users as there is less competition for resources on the macrocell. However, when a minimum throughput constraint is set, some users whose locations are within the small cells coverage cannot be offloaded to the small cell, and will be forced to remain on the macrocell. This increases competition for the macrocell bandwidth and reduces the average throughput of macro only users.

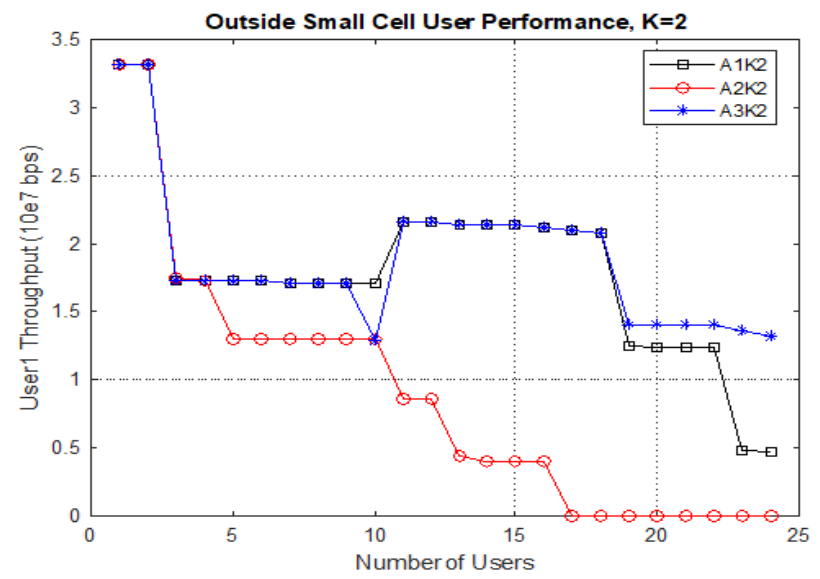

(a)

Figure 3. Throughput performance of Userl 


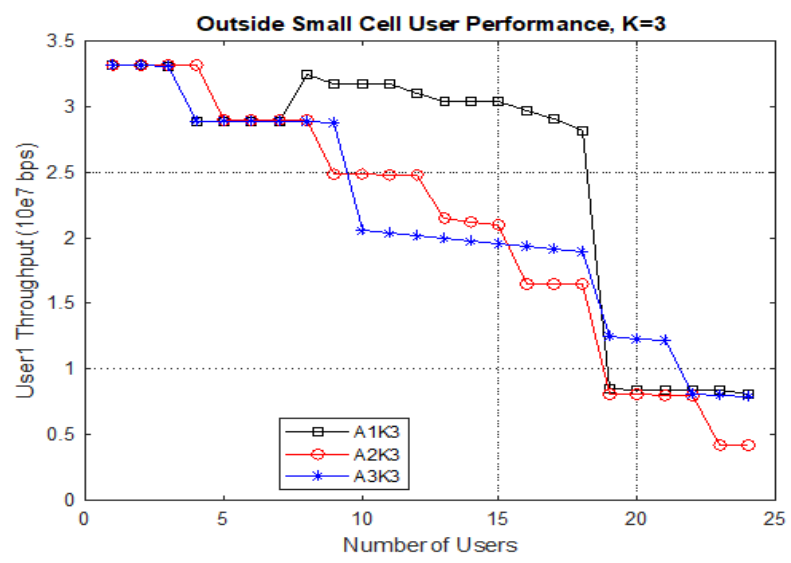

(b)

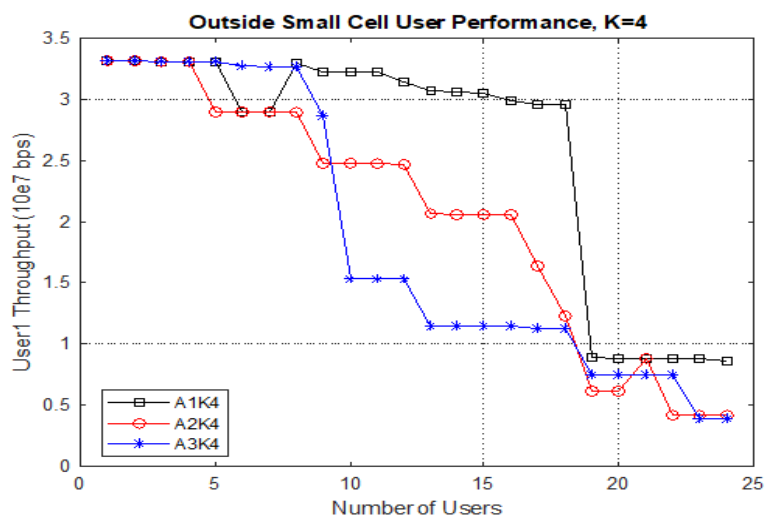

(c)

Figure 3. Throughput performance of Userl (continue)

\subsection{Small cell edge user performance (User2)}

Figures 4(a)-4(c) show the performance of a typical user at the edge of the small cell coverage (User2), with varying $K$ for A1, A2 and A3. User2's location is shown in Figure 1 . When $K=2$, User2 performance on A2 and A3 are comparable, with User2 experiencing between 3-5 Mbps as other users are added. In comparison, User 2 on $\mathrm{A} 1$ is dropped as early as when another 6 other users are added to the HetNet. At $K=3$, Figure 4(b) shows that A3 performs similar to A2. A3 is able to provide a more constant User2 throughput as the number of small cells is increased. Figure 4(c) shows that A2 outperforms A3.

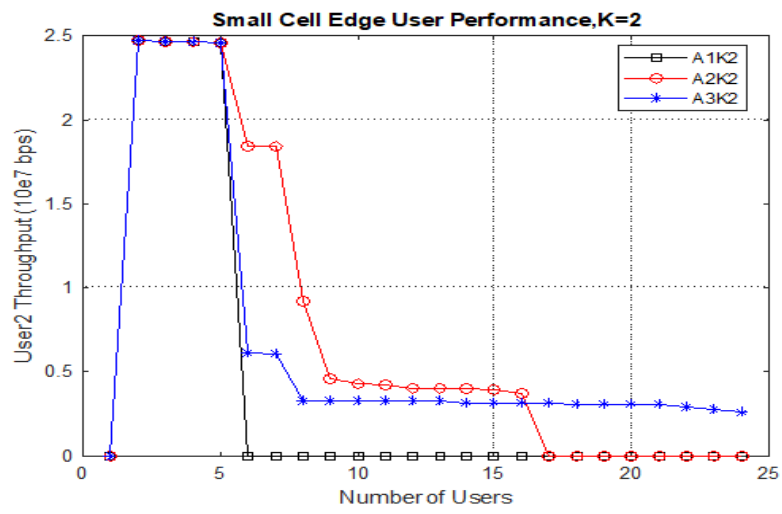

(a)

Figure 4. Throughput performance of user at edge of small cell coverage 


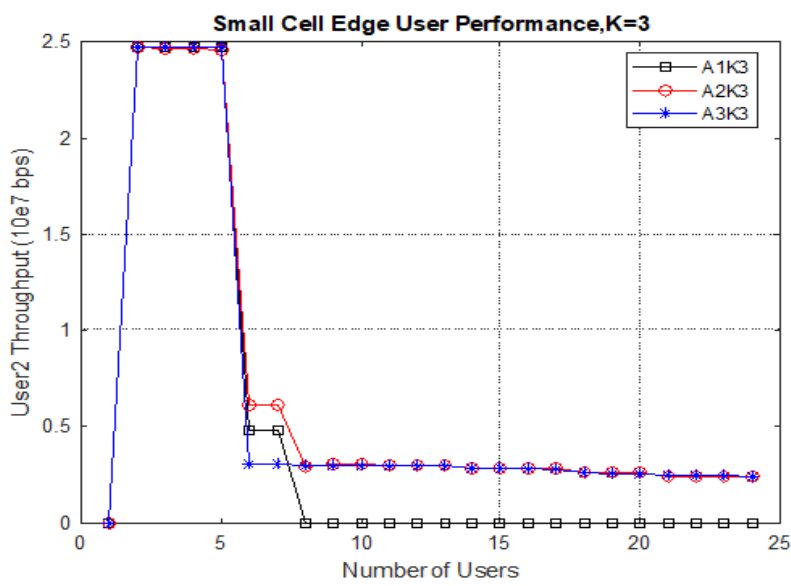

(b)

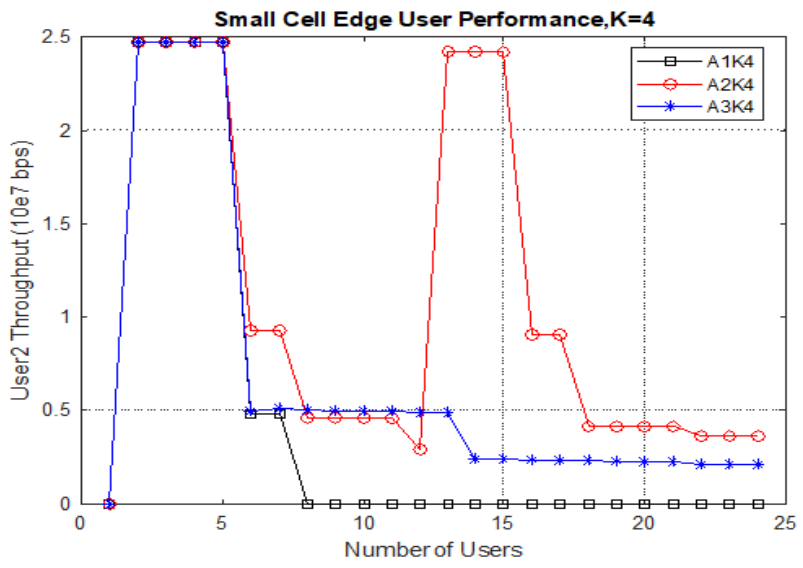

(c)

Figure 4. Throughput performance of user at edge of small cell coverage (continue)

Given the location of small cell edge users, these users can be allocated to any bordering cell, while the optimization process strives to maximize the HetNet capacity. Thus, as users are added, User2 performance fluctuates. A2 simply requires a minimum subband allocation as compared to A3 which requires a minimum throughput allocation. An example of the inconsistency in user throughput for A2 can be seen from the significant fluctuation in Figure 4(c) when there are 13-15 users in the HetNet. The results therefore show that for small cell edge users, the average user throughput is more consistent when using A3.

\subsection{Inside small cell user performance (User3)}

Figures 5(a)-5(c) show the performance of a typical user inside the coverage of both the macrocell and the small cell (User3), for A1, A2 and A3 for varying K. User3's location is shown in Figure 1. When $K=2$, A3 outperforms the other algorithms by providing User3 an average throughput of $8.98 \mathrm{Mbps}$. A1 drops User 3 while A2 provides the worst performance for User3 when only up to 8 other users are added to the HetNet. Figure 5(b) shows that A3 outperforms A1, but as the users are increased, A1 is able to provide better User3 average throughput. However, in Figure 5(c), A3 always outperforms A1, even as users increase. The above result summarizes that for users inside dual coverage, the A3 algorithm provides a mechanism to provide higher throughput and more stable user experience as the number of peer users are increased in the HetNet. 


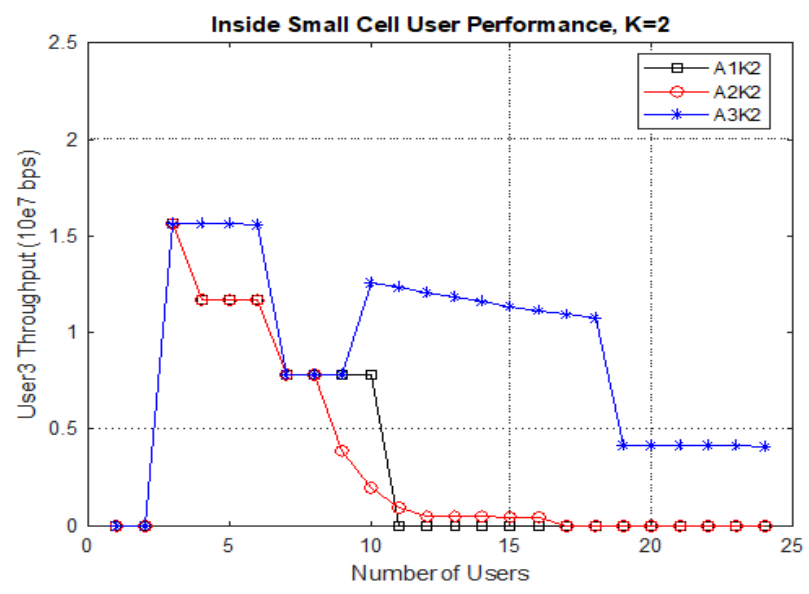

(a)

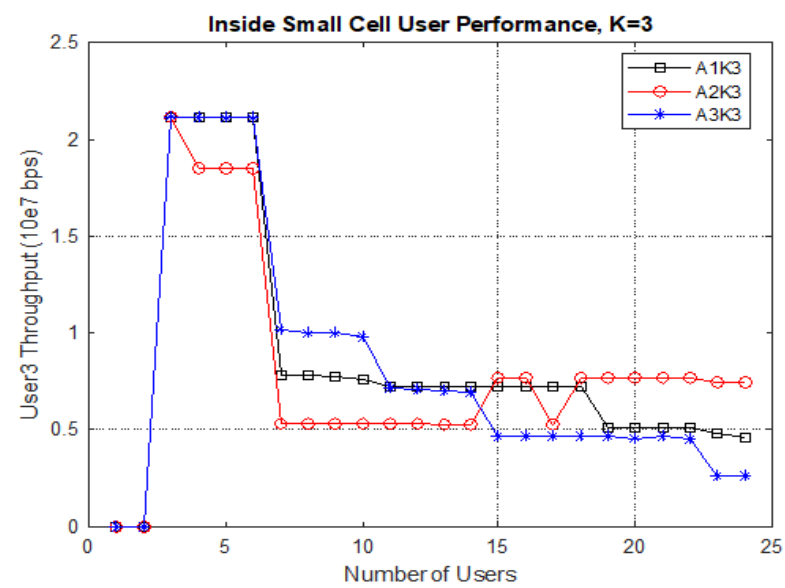

(b)

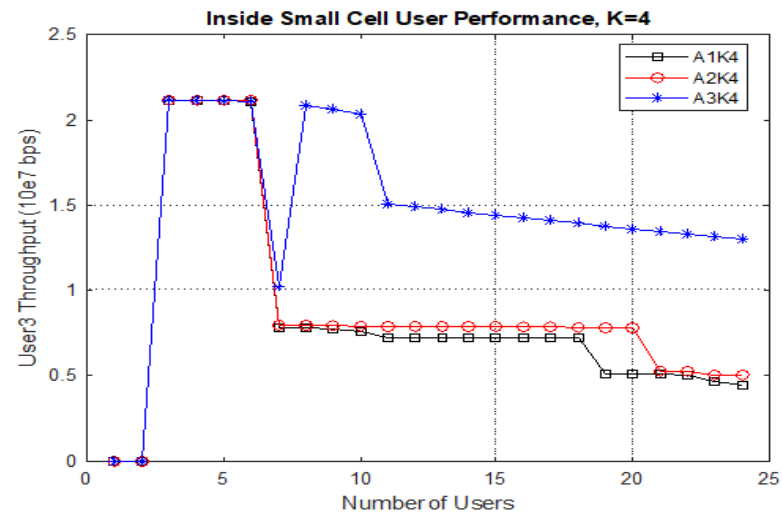

(c)

Figure 5. Throughput performance of user inside of small cell coverage

\subsection{Average user throughput performance}

Figures 6(a)-6(c) show the average user throughput for A1, A2 and A3 for varying $K$. Figure 6(a) shows that when $K=2$, the average user throughput for A1, A2 and A3 is $12.968 \mathrm{Mbps}, 7.038 \mathrm{Mbps}$ and $9.196 \mathrm{Mbps}$ respectively. Figure 6(b) shows that when $K=3$, the average user throughput for A1, A2 and A3 are 17.329 Mbps, 9. 644 Mbps and 11.286 Mbps respectively. Figure 6(c) shows that when $K=4$, the average user throughput for A1, A2 and A3 are 20.479 Mbps, 11.306 Mbps and 11.913 Mbps respectively. 
As expected, as the number of $K$ is increased, the average user throughput shows an increasing trend. This is due to increased resources available within the HetNet. Figure 6(a)-6(c) show that A1 outperforms A2 and A3 in terms of average user throughput performance inside the HetNet. However, it should be noted that the average user throughput shown is taken when considering the number of connected users. Thus, to understand the performance of $\mathrm{A} 1$ better, it is important to compare with the user drop rate shown in Figure 7. A2 is not compared in Figure 7 because the constraints of A2 force it to ensure each new user is allocated the minimum subband allocation, thus ensuring zero drop rate. A1K4 performance matches A1K3 performance, and is overlapping in Figure 7.

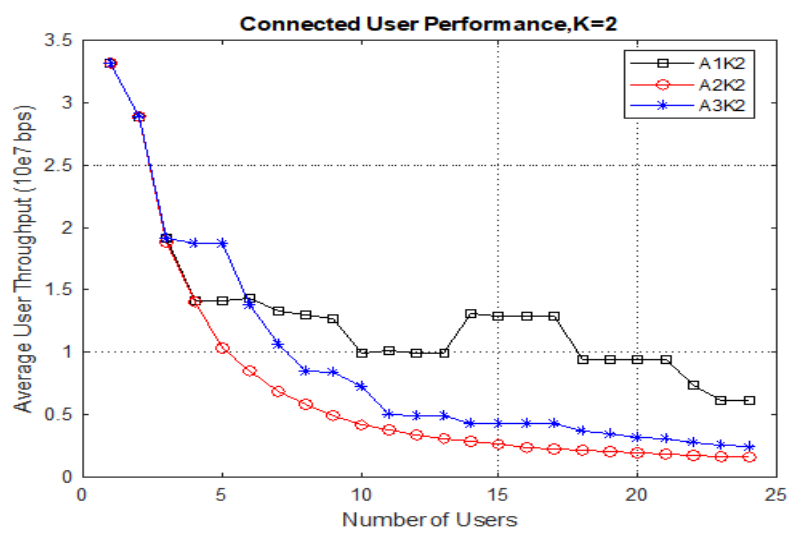

(a)

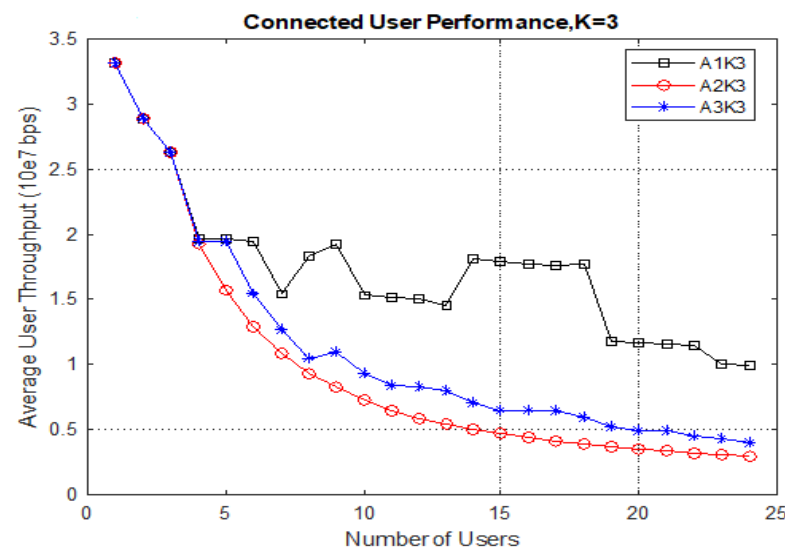

(b)

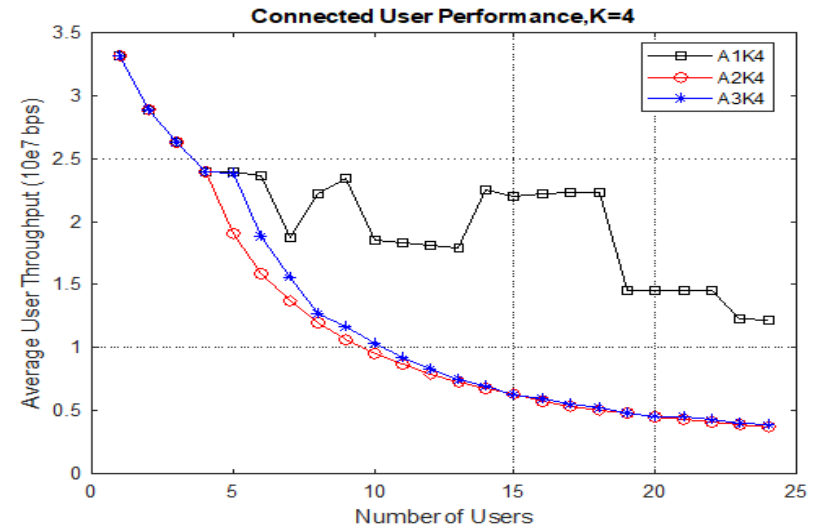

(c)

Figure 6. Average user throughput of HetNet 
In general, it is observed from Figure 7 that the drop rate reduces as the number of $K$ is increased for both A1 and A3. This is due to more available resources for each user to be provided the minimum requirements of the algorithms. A1 does not have any constraints, and thus, has a high drop rate of up to $80 \%$ because it only concerns itself with maximizing the capacity, without worrying about how many users it must sacrifice to achieve its goal. When $K=2$, A3 is at the highest drop rate of $45 \%$, while when $K=3$, A3 maximum drop rate is $35 \%$. A3's drop rate reduces dramatically to below $10 \%$ when $K=4$.

Figure 6(a)-6(c) also show that as $K$ increases, the performance of A3 performs marginally better than A2. The results therefore show that while both A2 and A3 can be used to enhance the average user throughput, A3 is able to also ensure that the user connection QoS is maintained via its minimum user throughput constraint. By comparing the throughput performance in Figure 6(a)-6(c) and the drop rate in Figure 7, A3 shows superiority in terms of ensuring the QoS of the user throughput and also the QoS of the HetNet in overall. A3 also demonstrates that a capacity maximization with a balanced utilization of all modes in a HetNet can be achieved without a bias setting for pico cells. These results suggest that the maximization of a HetNet capacity, with a controlled QoS and balanced load between macro and small cells can be achieved quickly, without having to set or optimize a bias setting for the small cell.

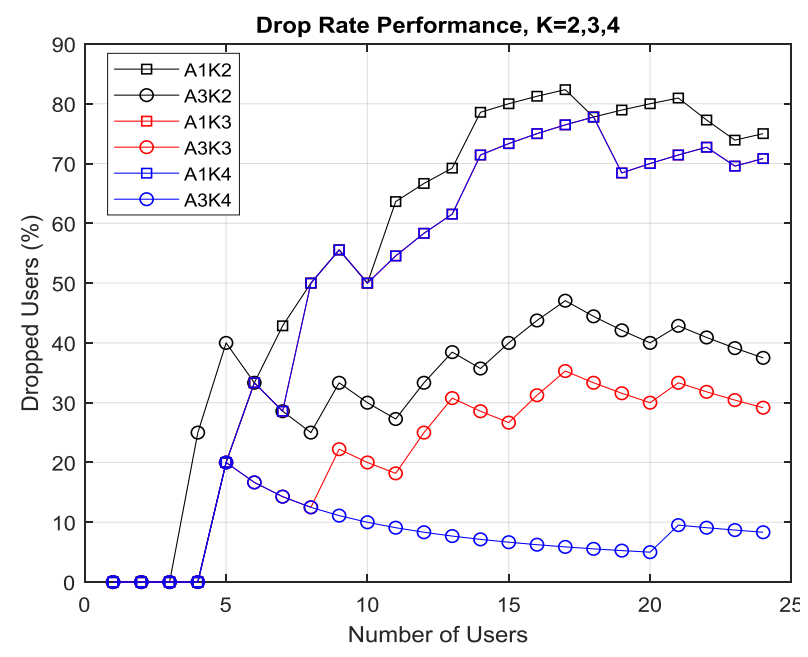

Figure 7. User drop rate comparison for A1 and A3

\section{CONCLUSION}

Three algorithms were evaluated to understand the user throughput performance in a HetNet network comprising of macro and small cells in an outdoor environment. The small cells were positioned at a determined distance away from the center of the macrocell, and the number of small cells were increased dynamically. Small cell biasing was not used to provide an accurate scenario of the SINR experienced in the HetNet. All algorithms controlled the user association to a given cell via a minimum power threshold. The optimization aimed to maximize the HetNet capacity while ensuring user and HetNet QoS. The results showed that the proposed scheme with a minimum user throughput constraint was able to control the QoS for the HetNet by maintaining a user drop rate of below 10\%, and the user drop rate reduced dramatically as the number of cells were increased.

\section{ACKNOWLEDGEMENTS}

This work is supported in part by the Fundamental Research Grant Scheme MMUE/150026.

\section{REFERENCES}

[1] Q. C. Li, H. Niu, A. T. Papathanassiou and G. Wu, "5G Network Capacity: Key Elements and Technologies," in IEEE Vehicular Technology Magazine, vol. 9, no. 1, pp. 71-78, March 2014.

[2] Yaqoob, Y. J. I. A., et al. "Performance Evaluation of Video Streaming on LTE With Coexistence of Wi-Fi Signal,” Bulletin of Electrical Engineering and Informatics, vol. 8, no. 3 pp. 890-889, Sep 2019. 
[3] Abdullah, Shapina, et al, "Design of a Cell Selection Mechanism to Mitigate Interference for Cell-Edge Macro Users in Femto-Macro Heterogeneous Network," Bulletin of Electrical Engineering and Informatics, vol. 8 no.1 pp. 180-187, March 2019.

[4] N. Trabelsi, C. S. Chen, R. El Azouzi, L. Roullet and E. Altman, "User Association and Resource Allocation Optimization in LTE Cellular Networks," in IEEE Transactions on Network and Service Management, vol. 14, no. 2, pp. 429-440, June 2017.

[5] Wang, Tong, et al. , "A Comprehensive Survey on Mobile Data Offloading in Heterogeneous Network," Wireless Networks, vol. 25, no. 2 pp. 573-584, 2019.

[6] Faizan Qamar, M. H. D. Nour Hindia, et al, "Interference Management Issues for the Future 5G Network: A Review," Telecommunication Systems, vol. 71, no.4, pp. 627-643, 2019.

[7] W. Tang, R. Zhang, Y. Liu and S. Feng, "Joint resource allocation for eICIC in heterogeneous networks," IEEE Global Communications Conference, Austin, TX, pp. 2011-2016, 2014.

[8] Q. Ye, B. Rong, Y. Chen, M. Al-Shalash, C. Caramanis and J. G. Andrews, "User Association for Load Balancing in Heterogeneous Cellular Networks," in IEEE Transactions on Wireless Communications, vol. 12, no. 6, pp. 2706-2716, June 2013.

[9] Y. Sun, D. W. K. Ng, J. Zhu and R. Schober, "Multi-Objective Optimization for Robust Power Efficient and Secure Full-Duplex Wireless Communication Systems," in IEEE Transactions on Wireless Communications, vol. 15 , no. 8 , pp. 5511-5526, Aug 2016.

[10] D. Fooladivanda and C. Rosenberg, "Joint Resource Allocation and User Association for Heterogeneous Wireless Cellular Networks," in IEEE Transactions on Wireless Communications, vol. 12, no. 1, pp. 248-257, January 2013.

[11] M. Ismail, W. Zhuang and M. Yu, "Radio Resource Allocation for Single-Network and Multi-Homing Services in Heterogeneous Wireless Access Medium,” 2012 IEEE Vehicular Technology Conference (VTC Fall), Quebec City, QC, pp. 1-5, 2012.

[12] Y. Gao, W. Jiang and T. Kaiser, "Bidirectional branch and bound based antenna selection in massive MIMO systems," 2015 IEEE 26th Annual International Symposium on Personal, Indoor, and Mobile Radio Communications (PIMRC), Hong Kong, pp. 563-568, 2015.

[13] Z. Li, S. Guo, D. Zeng, A. Barnawi and I. Stojmenovic, "Joint Resource Allocation for Max-Min Throughput in Multicell Networks," in IEEE Transactions on Vehicular Technology, vol. 63, no. 9, pp. 4546-4559, Nov. 2014.

[14] O. Oguejiofor and L. Zhang, "Global optimization of weighted sum-rate for downlink heterogeneous cellular networks," 2016 23rd International Conference on Telecommunications (ICT), Thessaloniki, pp. 1-6, 2016.

[15] Y. Shen, Y. Shi, J. Zhang and K. B. Letaief, "LORM: Learning to Optimize for Resource Management in Wireless Networks With Few Training Samples," in IEEE Transactions on Wireless Communications, vol. 19, no. 1, pp. 665-679, Jan. 2020.

[16] Yanzan Sun, Tianle Deng, Yong Fang, Min Wang and Yating Wu, "A method for pico-specific upper bound CRE bias setting in HetNet," 2013 IEEE Wireless Communications and Networking Conference Workshops (WCNCW), Shanghai, pp. 80-84, 2013.

[17] J. G. Andrews, S. Singh, Q. Ye, X. Lin and H. S. Dhillon, "An overview of load balancing in hetnets: old myths and open problems," in IEEE Wireless Communications, vol. 21, no. 2, pp. 18-25, April 2014.

[18] S. Nathaniel, S. H. S. Ariffin, A. Farzamnia and A. J. Adegboyega, "Multi-criteria load balancing decision algorithm for LTE network," 4th International Conference on Engineering Technology and Technopreneuship (ICE2T), Kuala Lumpur, pp. 57-62, 2014.

[19] Muñoz-Gea, et al, "Optimizatio Framework for Uplink Video Transmission in HetNets," Proceedings of Workshop on Mobile Video Delivery, no. 6, pp. 1-6, March 2014.

[20] A. Sayenko, M. Zolotukhin and T. Hämälainen, "On optimal placement of low power nodes for improved performance in heterogeneous networks," NOMS 2016 - 2016 IEEE/IFIP Network Operations and Management Symposium, Istanbul, pp. 349-357, 2016.

[21] Y. Sun, G. Feng, S. Qin, S. Sun and L. Zhang, "User Behavior Aware Cell Association in Heterogeneous Cellular Networks," 2017 IEEE Wireless Communications and Networking Conference (WCNC), San Francisco, CA, pp. 1-6, 2017.

[22] Carreras, A., et al., "A System-Level Simulator for the Downlink of LTE-A: Case of study-Cell-offloading in HetNets," Wireless Personal Communications, vol. 100, no. 1 pp. 177-191, 2018.

[23] S. Hu, X. Wang and M. Z. Shakir, "A MIH and SDN-based Framework for network selection in 5G HetNet: Backhaul requirement perspectives,” 2015 IEEE International Conference on Communication Workshop (ICCW), London, pp. 37-43, 2015.

[24] 3GPP, "Evolved Universal Terrestrial Radio Access (E-UTRA); Further advancements for E-UTRA physical layer aspects," Technical Specification (TS) 36.814, 3rd Generation Partnership Project (3GPP), Version 9.2.0, 2017.

[25] M. N. B. El-Haran, W. Tan, A. A. El-Saleh and S. P. Thiagarajah, "Joint Subchannel and Power Allocation Optimization in Heterogeneous Networks," 2018 International Conference on Advances in Computing, Communications and Informatics (ICACCI), Bangalore, pp. 1932-1937, 2018. 


\section{BIOGRAPHIES OF AUTHORS}
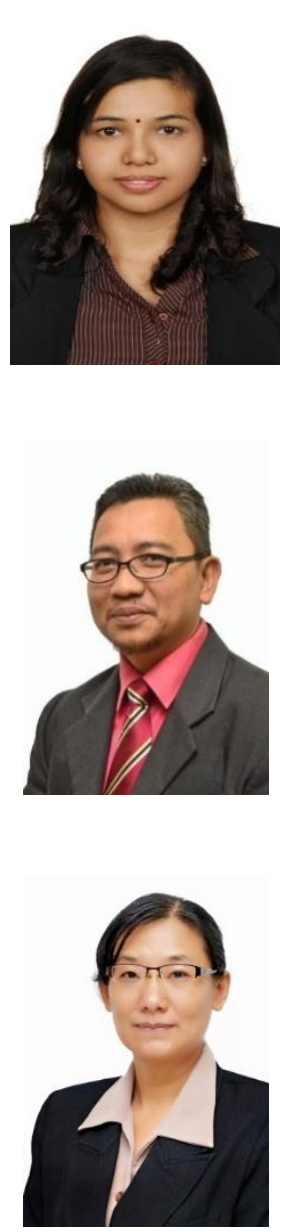

Siva Priya Thiagarajah is Lecturer at the Faculty of Engineering, Multimedia University (MMU). She received a Bachelor in Electrical Engineering (Telecommunications-Hons) from Technology University of Malaysia and Master of Engineering (Telecommunications) from MMU. She is currently pursuing her Ph.D. in Engineering in MMU. She is a Professional Engineer with Practicing Certificate with Board of Engineers Malaysia and a Corporate Member with the Institution of Engineers Malaysia. She is also Advisor to the MMU-Institute of Engineers Malaysia (IEM) Student Section. Prior to joining the academia, she worked in interference resolution, spectrum management and satellite procurement. Her research interests include satellite communications, wireless channel modelling/ optimization, IoT, and biomedical applications.

Mohamad Yusoff Alias is Professor at the Faculty of Engineering, MMU. He has been the Dean of Institute for Postgraduate Studies (IPS) since May 2014 and is a member of the university Senate. He received his Ph.D. in December 2004 from the School of Electronics and Computer Science (ECS), University of Southampton in the United Kingdom. He obtained a Bachelor of Science in Engineering (Electrical Engineering) degree from the University of Michigan in 1998. He is also the Counselor for Institute for Electrical and Electronics Engineer (IEEE) MMU Student Branch which recently won Region 10 (Asia \& Pacific) Exemplary Student Branch Award and 2018 R10 Student Branch Award. His research interests cover the field of wireless communications especially in OFDM, multiple antenna system, multiuser detection, genetic algorithms in communications, multimedia applications and visible light communications.

Wooi-Nee Tan is Lecturer at the Faculty of Engineering, MMU. She received a Bachelor in Computer Science and Education (Hons), Master of Science (Mathematics) and a Ph.D. degree in Mathematics from the Technology University of Malaysia. Her research interests include mathematical modeling of dynamical systems and optimization, demand side optimization and smart grid. 